\title{
AN EXTENSION THEOREM FOR MEROMORPHIC FUNCTIONS OF SEVERAL VARIABLES
}

\author{
JUHANI RIIHENTAUS
}

\section{Introduction}

1.1. Shiffman [4, Lemma 3] has obtained the following result concerning removable singularities of analytic functions of several complex variables.

Let $G$ be a domain of $C^{n}$. Let $E \subset G$ be closed in $G$ and of zero $(2 n-2)$-dimensional Hausdorff measure. Then each function $f$ analytic in $G \backslash E$ has a unique analytic extension to $G$.

Harvey-Polking [2, Remark after Theorem 1.1] announces that in view of the fact that the envelope of meromorphy is the same as the envelope of holomorphy the above result of Shiffman implies a similar result for meromorphic functions.

In [3, Theorem 3.5] Shiffman's result is generalized in the following way.

Let $G$ be a domain of $C^{n}$. Let $E \subset G$ be closed in $G$ and of $\sigma$-finite $(2 n-1)$ dimensional Hausdorff measure. Let $E_{1} \subset E$ (not necessarily closed in $G$ ) be of zero $(2 n-2)$-dimensional Hausdorff measure. Let $f: G \backslash E_{1} \rightarrow C$ be a continuous function such that $f$ is analytic in $G \backslash E$. Then $f$ has a unique analytic extension to $G$.

The purpose of this paper is to give a generalization to the above result of Harvey-Polking. Our generalization (Theorem 3.1 below) is analogous to the above generalization of Shiffman's result.

Our method of proof is to use our result above, Federer's and Shiffman's measure theoretic results and Levi's extension theorem.

1.2. We use mainly the same notation and terminology as in [3]. Moreover, the following notation is used.

Besides $C$ with its usual euclidean metric we also consider its one point compactification $\boldsymbol{C}^{*}=\boldsymbol{C} \cup\{\infty\}$ with the spherical metric:

$$
q(a, b)=\frac{|a-b|}{\left(\left(1+|a|^{2}\right)\left(1+|b|^{2}\right)\right)^{1 / 2}},
$$




$$
q(a, \infty)=\frac{1}{\left(1+|a|^{2}\right)^{1 / 2}},
$$

when $a, b \in \boldsymbol{C}$. The metric $q$ defines in $\boldsymbol{C}$ its usual euclidean topology.

Let $D$ be a subset of $C^{n}$. A function $f: D \rightarrow C^{*}$ is said to be spherically continuous if it is continuous when $C^{*}$ is equipped with the spherical metric $q$. Recall that a function $f: D \rightarrow C$ is spherically continuous if and only if it is continuous.

For the theory of analytic and meromorphic functions of several complex variables we refer to [6]. However, for the sake of completeness we recall here the following facts.

Let $G$ be a domain of $C^{n}$. Let $f$ be a function meromorphic in $G$. Then there is $N(f) \subset G$ closed in $G$ and $I(f) \subset N(f)$ closed in $G$ such that $f$ is analytic in $G \backslash N(f)$ and has a spherically continuous extension to $G \backslash I(f)$. Moreover, $N(f)$ is of $\sigma$-finite $(2 n-2)$-dimensional Hausdorff measure and $I(f)$ is of $\sigma$-finite $(2 n-4)$-dimensional Hausdorff measure (see e.g. [5, pp. 13-15]).

\section{Lemmas}

We begin with the following result due to Federer [1] and Shiffman; see [4, Corollary 4 and Lemma 2].

2.1. Lemma. Let $A$ be a Borel subset of $\boldsymbol{C}^{n}$. Let $\alpha$ be an arbitrary non-negative number and let $k$ be an integer such that $0 \leqq k \leqq n-1$.

(1) If $H^{2 n-2}(A)<\sigma_{\infty}$, then $H^{0}\left(A \cap p^{-1}\{x\}\right)<\sigma_{\infty}$ for $H^{2 n-2}$-almost all $x \in C^{n-1}$.

(2) If $H^{2 n-2}(A)=0$, then $A \cap p^{-1}\{x\}=\emptyset$ for $H^{2 n-2}$-almost all $x \in C^{n-1}$.

(3) If $a \in C^{n}, \alpha>0$ and $H^{2 k+\alpha}(A)=0$, then there is a complex $(n-k)$-plane $P$ through the point a such that $H^{\alpha}(A \cap P)=0$.

We recall that for an arbitrary set $B \subset C^{n}, H^{0}(B)$ is the number of points of $B$.

Next we give the properties of analytic and meromorphic functions we need in Section 3. For the first lemma see [3, Theorem 3.5].

2.2. Lemma. Let $G$ be a domain of $C^{n}$. Let $E \subset G$ be closed in $G$ and let $H^{2 n-1}(E)<\sigma_{\infty}$. Let $E_{1} \subset E$ be such that $H^{2 n-2}\left(E_{1}\right)=0$. Let $f: G \backslash E_{1} \rightarrow C$ be a continuous function such that $f \mid G \backslash E$ is analytic. Then there is a unique analytic function $f^{*}: G \rightarrow C$ such that

$$
f^{*} \mid G \backslash E_{1}=f .
$$

The next lemma is an adaptation of Lemma 2.2 in the case $n=1$.

2.3. Lemma. Let $G$ be a domain of $C$. Let $E \subset G$ be closed in $G$ and let $H^{1}(E)<\sigma_{\infty}$. Let $f: G \backslash E \rightarrow C$ be an analytic function such that $f$ has a spherically continuous extension $f^{*}: G \rightarrow C^{*}$. Then $f^{*}$ is meromorphic in $G$. 
Proof. If $z_{0} \in G$ and $f^{*}\left(z_{0}\right) \neq \infty$, Lemma 2.2 implies that $f^{*}$ is analytic in a neighborhood of $z_{0}$. If $f^{*}\left(z_{0}\right)=\infty$, it similarly follows that $1 / f^{*}$ is analytic in a neighborhood of $z_{0}$.

Our last lemma is Levi's extension theorem for meromorphic functions. See e.g. [2, Theorem $2.1(\mathrm{~b})]$.

2.4. Lemma. Let $z_{0}=\left(x_{0} ; y_{0}\right) \in C^{n}$ have a neighborhood $D^{n}\left(z_{0}, r\right)=D^{n-1}\left(x_{0}, s\right) \times$ $B^{2}\left(y_{0}, t\right)$. Let $t^{\prime}$ be such that $0<t^{\prime}<t$. Let $f: D^{n-1}\left(x_{0}, s\right) \times\left(B^{2}\left(y_{0}, t\right) \backslash \bar{B}^{2}\left(y_{0}, t^{\prime}\right)\right) \rightarrow C$ be an analytic function such that for $H^{2 n-2}$-almost all $x \in D^{n-1}\left(x_{0}, s\right)$ the analytic function $g_{x}: B^{2}\left(y_{0}, t\right) \backslash \bar{B}^{2}\left(y_{0}, t^{\prime}\right) \rightarrow C$,

$$
g_{x}(y)=f(x ; y)
$$

has a meromorphic extension to $B^{2}\left(y_{0}, t\right)$. Then $f$ has a unique meromorphic extension to $D^{n}\left(z_{0}, r\right)$.

\section{Singularities}

The next theorem is the result of this paper. It is an analogue of [3, Theorem 3.5 (Lemma 2.2 above)] for meromorphic functions. However, we formulate it for analytic functions.

3.1. Theorem. Let $G$ be a domain of $C^{n}$. Let $E \subset G$ be closed in $G$ and let $H^{2 n-1}(E)<\sigma_{\infty}$. Let $E_{1} \subset E$ be such that $H^{2 n-2}\left(E_{1}\right)=0$. Let $f: G \backslash E_{1} \rightarrow C^{*}$ be $a$ spherically continuous function such that $f \mid G \backslash E$ is analytic. Then $f \mid G \backslash E$ has a unique meromorphic extension to $G$.

Proof. Since $H^{2 n-1}(E)<\sigma_{\infty}$, int $E=\emptyset$. Hence it is sufficient to show that each point $z_{0} \in E$ has a neighborhood $U_{z_{0}}$ in $G$ such that $f \mid U_{z_{0}} \backslash E$ has a meromorphic extension to $U_{z_{0}}$. Let $z_{0} \in E$ be arbitrarily given.

We first consider the case $z_{0} \in E \backslash E_{1}$. Suppose first that $f\left(z_{0}\right) \neq \infty$. Using the spherical continuity of $f$ at the point $z_{0}$ and Lemma 2.2 we find a neighborhood $U_{z_{0}}$ of $z_{0}$ in $G$ such that $f \mid U_{z_{0}} \backslash E$ has a unique analytic extension $g^{*}: U_{z_{0}} C \rightarrow$.

If $f\left(z_{0}\right)=\infty$, it similarly follows that there is a neighborhood $U_{z_{0}}$ of $z_{0}$ in $G$ such that $1 / f \mid U_{z_{0}} \backslash E$ has a unique analytic extension $h: U_{z_{0}} \rightarrow C$. Therefore $h^{*}=1 / h: U_{z_{0}} \rightarrow C^{*}$ gives the desired meromorphic extension of $f \mid U_{z_{0}} \backslash E$ to $U_{z_{0}}$. Moreover, $h^{*}$ is spherically continuous.

To treat the case $z_{0}=\left(x_{0} ; y_{0}\right) \in E_{1}$ we proceed as follows.

Denote

$$
E_{1}^{\prime}=\left(G \backslash \bigcup_{z \in E \backslash E_{1}} U_{z}\right) \cap E,
$$


where $U_{z}$ is as above. Then $E_{1}^{\prime} \subset G$ is closed in $G$. Since $E_{1}^{\prime} \subset E_{1}, H^{2 n-2}\left(E_{1}^{\prime}\right)=0$. Define $f^{*}: G \backslash E_{1}^{\prime} \rightarrow C^{*}$,

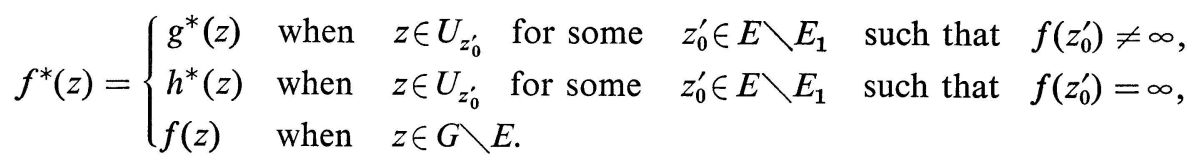

Since int $E=\emptyset, f^{*}$ is well defined. Moreover, $f^{*}$ is meromorphic and spherically continuous. Therefore there is a set $N\left(f^{*}\right) \subset G \backslash E_{1}^{\prime}$ closed in $G \backslash E_{1}^{\prime}$ such that $H^{2 n-2}\left(N\left(f^{*}\right)\right)<\sigma_{\infty}$ and $f^{*} \mid\left(G \backslash E_{1}^{\prime}\right) \backslash N\left(f^{*}\right)$ is analytic. Denote $E_{1}^{\prime \prime}=E_{1}^{\prime} \cup N\left(f^{*}\right)$. Then $E_{1}^{\prime \prime} \subset G$ is closed in $G$ and $H^{2 n-2}\left(E_{1}^{\prime \prime}\right)<\sigma_{\infty}$. By Lemma 2.1 (3) there is a complex plane $P$ through the point $z_{0}$ such that $H^{1}\left(E_{1}^{\prime \prime} \cap P\right)=0$. Rotating the coordinate system, if necessary, we may assume $P=\left\{x_{0}\right\} \times C$.

Since $H^{1}\left(E_{1}^{\prime \prime} \cap\left(\left\{x_{0}\right\} \times C\right)\right)=0$, there is $t>0$ such that $\left\{x_{0}\right\} \times \bar{B}^{2}\left(y_{0}, t\right) \subset G$ and $\left\{x_{0}\right\} \times S^{1}\left(y_{0}, t\right) \subset G \backslash E_{1}^{\prime \prime}$. Since $\left\{x_{0}\right\} \times S^{1}\left(y_{0}, t\right)$ is compact in $G \backslash E_{1}^{\prime \prime}$ and $E_{1}^{\prime \prime}$ is closed in $G$, there are $s=\left(r_{1}, \ldots, r_{n-1}\right), r_{j}>0, j=1, \ldots, n-1$, and $t_{1}, t_{2}, 0<t_{1}<t<t_{2}$, such that $D^{n-1}\left(x_{0}, s\right) \times B^{2}\left(y_{0}, t_{2}\right) \subset G$ and $D^{n-1}\left(x_{0}, s\right) \times\left(B^{2}\left(y_{0}, t_{2}\right) \backslash \bar{B}^{2}\left(y_{0}, t_{1}\right)\right) \subset$ $G \backslash E_{1}^{\prime \prime}$. Hence $f^{*} \mid D^{n-1}\left(x_{0}, s\right) \times\left(B^{2}\left(y_{0}, t_{2}\right) \backslash \bar{B}^{2}\left(y_{0}, t_{1}\right)\right)$ is analytic.

Denote

$$
\begin{aligned}
& B_{1}=\left\{x \in D^{n-1}\left(x_{0}, s\right) \mid H^{0}\left(\left(\{x\} \times B^{2}\left(y_{0}, t_{2}\right)\right) \cap N\left(f^{*}\right)\right)<\sigma_{\infty}\right\}, \\
& B_{2}=\left\{x \in D^{n-1}\left(x_{0}, s\right) \mid\left(\{x\} \times B^{2}\left(y_{0}, t_{2}\right)\right) \cap E_{1}^{\prime}=\emptyset\right\}, \\
& B=B_{1} \cap B_{2} .
\end{aligned}
$$

By Lemma 2.1 (1) and (2), $H^{2 n-2}\left(D^{n-1}\left(x_{0}, s\right) \backslash B\right)=0$. For each $x \in B$ denote

$$
E_{n}(x)=\left\{y \in B^{2}\left(y_{0}, t_{2}\right) \mid(x ; y) \in N\left(f^{*}\right)\right\} .
$$

Then $E_{n}(x) \subset B^{2}\left(y_{0}, t_{2}\right)$ is closed in $B^{2}\left(y_{0}, t_{2}\right)$ and $H^{0}\left(E_{n}(x)\right)<\sigma_{\infty}$. For each $x \in B$ define $g_{x}: B^{2}\left(y_{0}, t_{2}\right) \rightarrow C^{*}$,

$$
g_{x}(y)=f^{*}(x ; y) .
$$

Then $g_{x}$ is spherically continuous and $g_{x} \mid B^{2}\left(y_{0}, t_{2}\right) \backslash E_{n}(x)$ is analytic. By Lemma 2.3 $g_{x}$ is meromorphic.

By Lemma $2.4 f^{*} \mid D^{n-1}\left(x_{0}, s\right) \times\left(B^{2}\left(y_{0}, t_{2}\right) \backslash \bar{B}^{2}\left(y_{0}, t_{1}\right)\right)$ has a unique meromorphic extension to $D^{n-1}\left(x_{0}, s\right) \times B^{2}\left(y_{0}, t_{2}\right)$, which concludes the proof.

3.2. Corollary. Let $G$ be a domain of $C^{n}$. Let $E \subset G$ be closed in $G$ and let $H^{2 n-2}(E)=0$. Let $f$ be meromorphic in $G \backslash E$. Then $f$ has a unique meromorphic extension to $G$.

Proof. Since $f$ is meromorphic in $G \backslash E$, there is a set $N(f) \subset G \backslash E$ closed in $G \backslash E$ such that $H^{2 n-2}(N(f))<\sigma_{\infty}$ and $f$ is analytic in $(G \backslash E) \backslash N(f)$. Similarly, there is a set $I(f) \subset N(f)$ such that $H^{2 n-2}(I(f))=0$ and $f \mid(G \backslash E) \backslash N(f)$ has a spherically continuous extension to $(G \backslash E) \backslash I(f)$. Denote $F=E \cup N(f)$ and $F_{1}=E \cup I(f)$. Then $F \subset G$ is closed in $G$ and $H^{2 n-1}(F)<\sigma_{\infty}$. Similarly, $F_{1} \subset F$ 
and $H^{2 n-2}\left(F_{1}\right)=0$. Moreover, the analytic function $f \mid G \backslash F$ has a spherically continuous extension to $G \backslash F_{1}$. Hence the assertion follows from Theorem 3.1.

3.3. Remark. The result of Theorem 3.1 is sharp in the following sense: If we assume that $H^{2 n-2}\left(E_{1}\right)=0$, then the condition $H^{2 n-1}(E)<\sigma_{\infty}$ cannot for any $\alpha>0$ be replaced by the condition $H^{2 n-1+\alpha}(E)=0$. This can be seen from the example given in [3, Remark 3.4].

3.4. Remark. The result of Theorem 3.1 is sharp also in the following sense: If we assume that $H^{2 n-1}(E)<\sigma_{\infty}$, then the condition $H^{2 n-2}\left(E_{1}\right)=0$ cannot be replaced by the condition $H^{2 n-2}\left(E_{1}\right)<\infty$. This can be seen from the following example.

The function $f: D^{n} \backslash\left(D^{n-1} \times\{0\}\right) \rightarrow C$,

$$
f(z)=e^{1 / y},
$$

is analytic. Here $E=E_{1}=D^{n-1} \times\{0\}$ and thus $H^{2 n-2}\left(E_{1}\right)<\infty$. However, $f$ has no meromorphic extension to $D^{n}$.

\section{References}

[1] Federer, H.: Geometric measure theory. - Springer-Verlag, Berlin-Heidelberg-New York, 1969.

[2] Harvey, R., and J. Polking: Extending analytic objects. - Comm. Pure Appl. Math. 28, 1975, $701-727$.

[3] Rinhentaus, J.: Removable singularities of analytic functions of several complex variables. Math. Z. 158, 1978, 45-54.

[4] Shiffman, B.: On the removal of singularities of analytic sets. - Michigan Math. J. 15, 1968, $111-120$.

[5] Stolzenberg, G.: Volumes, limits, and extensions of analytic varieties. - Lecture Notes in Mathematics 19, Springer-Verlag, Berlin-Heidelberg-New York, 1966.

[6] Whitney, H.: Complex analytic varieties. - Addison-Wesley, Reading-Menlo Park-London-Don Mills, 1972.

Kosteperänkatu 2 B 84

SF-90100 Oulu 10

Finland

Received 15 March 1978 